\title{
Efeito do teor de zircônio e da deformação no envelhecimento de ligas Al-Zr
}

\author{
Paulo Mendel Fernandes ${ }^{1 *}$ (1) \\ Carlos Augusto Silva de Oliveira ${ }^{1}$
}

\begin{abstract}
Resumo
Esse trabalho teve o objetivo de estudar o efeito do teor de zircônio e da deformação na precipitação de ligas Al-Zr (Al-0,2\%p.Zr e Al-0,32\%p.Zr) durante envelhecimento. As ligas foram obtidas a partir do resfriamento rápido do metal líquido em molde de cobre resfriado com água; posteriormente as amostras foram cortadas, forjadas $(50$ e $70 \%$ de redução de altura) e envelhecidas a $377{ }^{\circ} \mathrm{C}$ por $8,12,50,100$ e 400 horas. As microestruturas foram analisadas por microscopia eletrônica de transmissão (MET) e as propriedades foram analisadas por resistividade elétrica e microdureza. As ligas indicaram um pico de endurecimento com 100 horas de envelhecimento devido à precipitação de intermetálicos $\mathrm{Al}_{3} \mathrm{Zr}$ cúbicos, após 400 horas as ligas apresentaram redução da microdureza devido ao superenvelhecimento. Tanto a deformação quanto o teor de zircônio influenciaram na precipitação, contudo, foi observado que o aumento da concentração de $\mathrm{Zr}$ foi mais significativo para o aumento na precipitação e, consequentemente, aumento do endurecimento das ligas Al-Zr.
\end{abstract}

Palavras-chave: Ligas Al-Zr; Deformação; Envelhecimento; Precipitação.

\section{Effect of zircon content and deformation on aging of the Al-Zr alloys}

\begin{abstract}
This work aimed to study the effect of zirconium content and deformation on the precipitation of $\mathrm{Al}-\mathrm{Zr}$ alloys (Al-0.2\%wt.Zr and Al-0.32\%wt.Zr) during aging. The alloys were obtained from the rapid cooling of the liquid metal in a water-cooled copper mold; after that, the samples were cut, forged (50 and $70 \%$ height reduction) and aged at $377^{\circ} \mathrm{C}$ for 8 , $12,50,100$ and 400 hours. The microstructures were analyzed by transmission electron microscopy (MET) and the properties were analyzed by electrical resistivity and microhardness. The alloys indicated a hardening peak with 100 hours of aging due to the precipitation of cubic $\mathrm{Al}_{3} \mathrm{Zr}$ intermetallic, after 400 hours the alloys showed reduction of the microhardness due to superaging. Both the deformation and the zirconium content influenced the precipitation, however, it was observed that the increase of the $\mathrm{Zr}$ concentration was more significant for the increase the precipitation and, consequently, increase the hardening of the Al-Zr alloys.
\end{abstract}

Keywords: Al-Zr alloys; Deformation; Ageing; Precipitation.

\section{Introdução}

O alumínio possui propriedades relevantes para aplicação nos diversos setores industriais, no qual se destacam a elevada condutividade elétrica e térmica, baixa densidade, elevada resistência a corrosão e elevada resistência específica. Porém, a baixa resistência mecânica e o baixo ponto de fusão são características que limitam as aplicações desse material, principalmente para o setor automotivo e aeroespacial $[1,2]$.

As ligas de alumínio são mais resistentes mecanicamente que o alumínio puro e apresentam uma maior variedade de aplicações. As ligas de alumínio endurecíveis por precipitação se destacam pela resistência mecânica e baixo peso. Essas após tratamento térmico apresentam partículas de pequeno diâmetro distribuídas homogeneamente pela microestrutura [3]. As ligas Al-Zr se destacam nessa classe de materiais por formarem precipitados $\mathrm{Al}_{3} \mathrm{Zr}$ de estrutura cúbica na matriz de alumínio. Esses precipitados bloqueiam ou dificultam a movimentação de discordâncias, resultando em um endurecimento do material [4-10].

Os precipitados $\mathrm{Al}_{3} \mathrm{Zr}$ cúbicos apresentam coerência com a matriz de alumínio, baixa difusividade, baixa solubilidade e baixa taxa de coalescimento. A baixa cinética de crescimento dessas partículas, por exemplo, permite que

${ }^{\prime}$ Laboratório de Conformação Mecânica - LabConf, Universidade Federal de Santa Catarina - UFSC, Florianópolis, SC, Brasil.

*Autor correspondente: paulomendel000@gmail.com

2176-1523 C) 2021. Fernandes et al. Publicado pela ABM. Este é um artigo publicado em acesso aberto (Open Access) sob a licença Creative Commons Attribution, que permite uso, distribuição e reprodução em qualquer meio, sem restrições desde que o trabalho original seja corretamente citado. 
características como raio, distribuição e distância entre as mesmas sejam mantidos com o aumento de temperatura, resultando também na manutenção das propriedades da liga e permitindo a aplicação desses materiais na transmissão de energia por cabos (condutividade elétrica) [11-14]. Nessa aplicação as ligas Al-Zr apresentam vantagens, como: resistência mecânica, baixo peso e temperatura de uso de até $240^{\circ} \mathrm{C}$ [15].

A deformação plástica pode alterar as propriedades mecânicas e causar diversas modificações microestruturais nos metais, entre elas pode se destacar a mudança na forma dos grãos, o aumento na densidade de discordâncias e formação de regiões com deformação heterogênea (bandas de cisalhamento, bandas de deformação, etc.). Essas heterogeneidades formadas na microestrutura podem tanto facilitar a difusão de soluto quanto servir de substrato para formação dos precipitados (precipitação heterogênea) [16-18]. Nesse trabalho foi aplicada deformação plástica por recalque (forjamento). Essa deformação é responsável pela formação de três zonas distintas no material: zona morta ou sem deformação, zona de média deformação e zona de alta deformação [19].

Esse trabalho tem por objetivo avaliar a influência do teor de zircônio e da quantidade de deformação na precipitação dos intermetálicos $\mathrm{Al}_{3} \mathrm{Zr}$ em ligas $\mathrm{Al}-\mathrm{Zr}$ após envelhecimento a $377^{\circ} \mathrm{C}$ em função do tempo. Os resultados foram analisados por microscopia eletrônica de transmissão, resistividade elétrica e microdureza.

\section{Metodologia}

Foram utilizadas duas ligas peritéticas Al-Zr, uma hipoperitética (Al-0,2\%p.Zr) e outra hiperperitética (Al-0,32\%p.Zr), conforme composição química mostrada na Tabela 1. A determinação da composição química da liga Al-0,2\%p.Zr foi realizada em um espectrômetro óptico de marca BRUKER e modelo Q4 TASMAN, já para liga $0,32 \%$ p.Zr foi utilizado um espectrômetro de emissão óptica com plasma indutivamente acoplado, fabricante PERKIN ELMER, modelo OPTIMA 8300.

Os lingotes das ligas foram fundidos conforme descrito a seguir: primeiramente, o alumínio de alta pureza ( $>99.8 \%$ p.) foi adicionado em um cadinho de carbeto de silício (SiC), juntamente, com waffles de Al-10\%p.Zr. $\mathrm{O}$ cadinho foi aquecido em um forno mufla até $847^{\circ} \mathrm{C}$ e mantido nessa temperatura por 40 minutos, depois o metal líquido foi retirado do forno e desgaseificado com argônio. Posteriormente, o metal líquido foi vazado em um molde de cobre resfriado com água nas paredes e no fundo até a temperatura ambiente. Esse resfriamento rápido foi realizado

Tabela 1. Composição química em porcentagem em peso

\begin{tabular}{cccccc}
\hline Liga & $\mathbf{M g}$ & $\mathbf{Z r}$ & $\mathbf{F e}$ & $\mathbf{S i}$ & Al \\
\hline Al-0,20Zr & $<0,01$ & 0,23 & 0,10 & 0,05 & Restante \\
Al-0,32Zr & - & 0,32 & 0,10 & 0,03 & Restante \\
\hline
\end{tabular}

para se obter o Zr em solução sólida, obteve-se os lingotes das ligas Al-0,2\%p.Zr e Al-0,32\%p.Zr.

As amostras foram obtidas em formato de paralelepípedo (15 $\mathrm{mm}$ de comprimento, $10 \mathrm{~mm}$ de altura e $10 \mathrm{~mm}$ de largura) a partir do corte dos lingotes em uma serra de fita de marca ERGOP. As amostras foram recalcadas (forjamento) sob compressão em matriz aberta por uma prensa de fricção GUTMANN (capacidade de 150 toneladas e potência de 7,5 HP-horsepower). As amostras da liga Al-0,2\%p.Zr foram recalcadas com $50 \%$ de redução de altura e as amostras da liga Al-0,32\%p.Zr com 50 e 70\%. Após a deformação foi realizado o envelhecimento em um forno mufla JUNG, modelo 3012 , na temperatura de $377^{\circ} \mathrm{C} \pm 1{ }^{\circ} \mathrm{C}$ nos tempos de $8,12,50,100$ e 400 horas. Após o tratamento térmico, as amostras foram retiradas do forno e resfriadas em água até a temperatura ambiente $\left(25^{\circ} \mathrm{C}\right)$.

A microestrutura foi analisada em um MET JEOL JEM 1011 de 100 kV. As amostras foram lixadas (Disk Grinder) até uma espessura de $100 \mu \mathrm{m}$ e depois puncionadas (Disk Punch) para se obter discos de $3 \mathrm{~mm}$ de diâmetro. Esses discos passaram por um polimento eletrolítico em uma máquina de jateamento duplo STRUERS TENUPOL-5 com temperatura de $3 \pm 3{ }^{\circ} \mathrm{C}$, tensão de operação de $8 \mathrm{~V}$ e eletrólito constituído de $2 / 3$ de metanol e 1/3 de ácido nítrico. Depois do ataque, as amostras foram imersas em metanol três vezes e em seguida em álcool etílico por três vezes para eliminar os resíduos [20].

Os ensaios de resistividade elétrica foram realizados em um equipamento Model 197A Autoranging Microvolt DMM Instructional Manual pelo método das quatro pontas, utilizando Spray da marca Sprayon-Silimatic (aerosol) nos contatos com as amostras a fim de melhorar os resultados. Foram obtidas as medidas de resistência elétrica e posteriormente foi calculada a resistividade elétrica multiplicando a resistência elétrica pela área da seção transversal e dividindo o total pelo comprimento do corpo de prova [18]. Foram realizadas cinco repetições da resistência elétrica para cada condição estudada. As amostras das ligas tiveram dimensões média de $16 \mathrm{~mm}$ de comprimento, 2,7 $\mathrm{mm}$ de altura e 5,6 $\mathrm{mm}$ de largura.

A microdureza foi realizada após metalografia padrão com carga de 500 gf e tempos de identação de 10 segundos, segundo especificações da norma ASTM E384 (American Society for Testing and Materials). Foram realizadas 10 medidas de microdureza para cada amostra na região de média deformação [19].

\section{Resultados e discussão}

\subsection{Análise microestrutural}

A Figura 1 apresenta as microestruturas das amostras da liga Al-0,32\%p.Zr sem envelhecimento com 50\% de redução de altura (A), e (B e C) envelhecidas por 100 horas a 


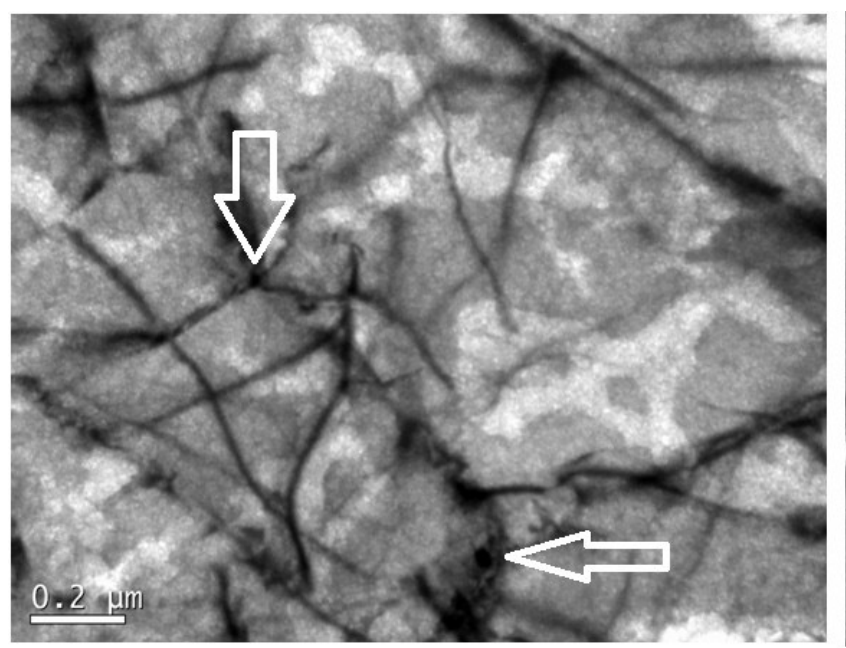

(A)

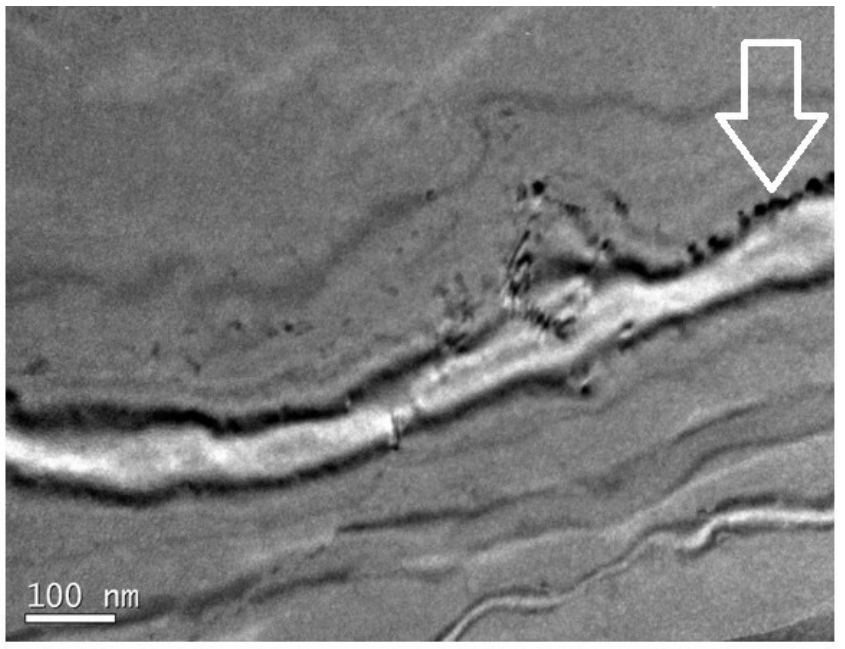

(B)

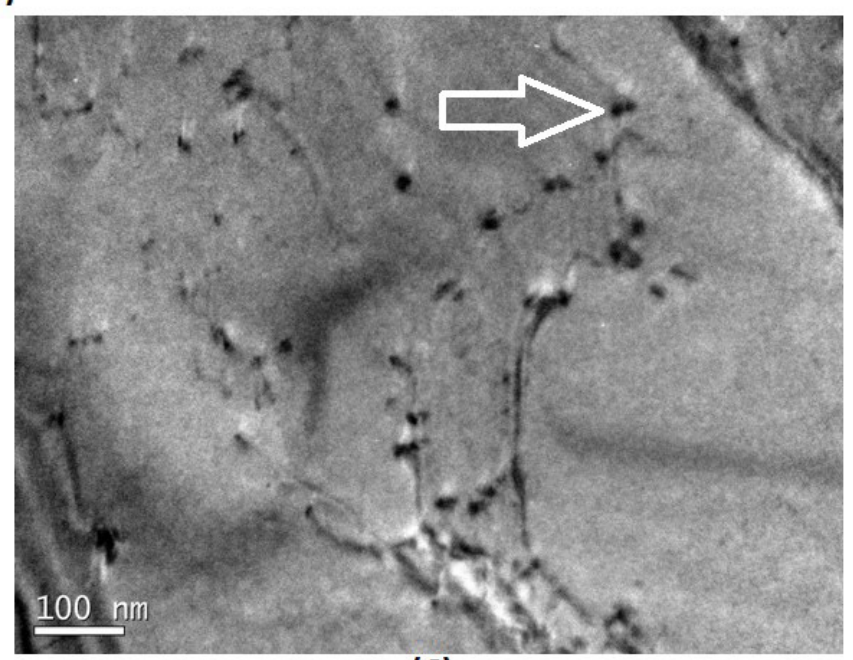

(C)

Figura 1. (A) Microestruturas das amostras da liga Al-0,32\%p.Zr deformada com 50\% de redução de altura sem envelhecimento e (B) e (C) com $70 \%$ de redução de altura envelhecida a $377^{\circ} \mathrm{C}$ por 100 horas. As setas indicam a presença de precipitados ao longo de discordâncias. Imagens obtidas por MET.

$377^{\circ} \mathrm{C} \operatorname{com} 70 \%$ de redução de altura. São indicadas (setas) a presença de precipitados nas discordâncias.

A liga de maior concentração em zircônio $(0,32 \%$ p.Zr) e maior grau de deformação (70\%) apresentou uma maior quantidade de precipitados $\mathrm{Al}_{3} \mathrm{Zr}$, o que indica a contribuição do aumento de concentração de zircônio e da deformação para aumentar a quantidade dessas partículas. A força motriz para precipitação é maior nas ligas com maior concentração em zircônio, de modo que favorece o aumento no número de precipitados [4-8]. As discordâncias geradas após deformação são sítios que facilitam a precipitação heterogênea pela microestrutura e também favorecem o aumento na quantidade de precipitados.

A fim de estudar a distribuição dos precipitados na microestrutura foi selecionado um PDAS (Padrão de difração de área selecionada), conforme Figura 2, de uma imagem de campo claro da amostra da liga Al- $0,32 \%$ p. $\mathrm{Zr}$ deformada em $70 \%$, de modo que a partir dessa foi utilizado a reflexão (111) para realizar uma varredura pela microestrutura obtendo imagens de campo claro e as respectivas imagens de campo escuro.

No PDAS são indicadas as reflexões (200), (100) e (111). A primeira reflexão é característica da matriz de alumínio, a segunda é referente ao precipitado $\mathrm{Al}_{3} \mathrm{Zr}$ cúbico e a última é comum as duas fases (precipitado e matriz) $[6,11]$. As microestruturas $\mathrm{a}, \mathrm{b}$ e $\mathrm{c}$ referem-se às diferentes regiões da liga, no qual pode ser observado uma distribuição heterogênea dos precipitados $\mathrm{Al}_{3} \mathrm{Zr}$ cúbicos (pontos brilhantes) com formas globulares e alongadas. Os precipitados $\mathrm{Al}_{3} \mathrm{Zr}$ cúbicos foram observados nos contornos e no interior dos grãos associados a heterogeneidades de deformação (discordâncias). Após envelhecimento por 100 horas essa quantidade de precipitados foi aumentada, principalmente, na liga com maior concentração de soluto e deformação. 


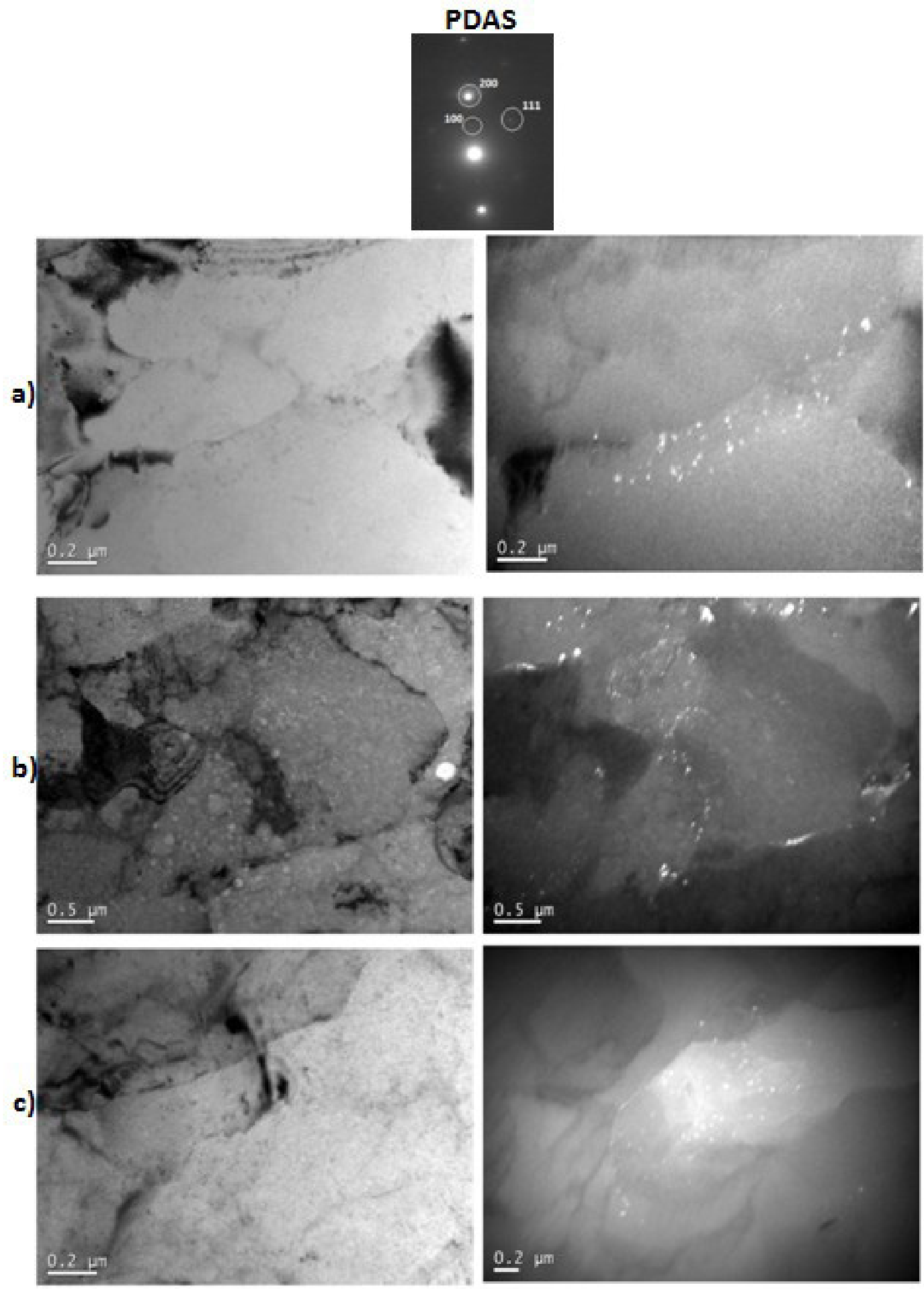

Figura 2. PDAS obtido do campo claro da amostra da liga Al-0,32\%p.Zr com $70 \%$ de redução de altura envelhecida por 100 horas a $377{ }^{\circ} \mathrm{C}$. a), b) e c) microestruturas observadas pela técnica de campo claro e escuro, respectivamente, obtidas a partir do PDAS mostrado utilizando a reflexão (111). 
Essa distribuição e variação na forma dos precipitados são devido à deformação plástica utilizada, no qual a mesma gerou sítios na microestrutura das amostras que facilitaram a precipitação e difusão de soluto resultando em uma concentração preferencial dessas partículas ao longo de regiões deformadas, discordâncias ou em contornos de grão. O coalescimento também pode ter contribuído para distribuição heterogênea destes, já que foram observados precipitados de diferentes tamanhos, de modo que os maiores possam crescer em função dos menores. Na Figura 1 A foram identificados precipitados na condição sem envelhecimento, de modo que estes possam crescer em função dos precipitados menores formados durante o envelhecimento $[12,14]$.

Souza [6] estudou as mesmas composições e condições de envelhecimento que o presente trabalho, porém não foi aplicada deformação. Foi observado uma distribuição homogênea dos precipitados $\mathrm{Al}_{3} \mathrm{Zr}$ pela microestrutura das ligas, em que esses apresentaram forma globular (equiaxial). Logo, os resultados observados por Souza [6] corroboram com os observados no presente trabalho no tocante a contribuição da deformação para precipitação, que favoreceu uma distribuição heterogênea dos precipitados $\mathrm{Al}_{3} \mathrm{Zr}$ e variação na forma dos mesmos.

\subsection{Resistividade elétrica}

A Figura 3 apresenta a variação da resistividade elétrica para as três condições estudadas. É possível observar que essas apresentaram redução da resistividade entre $12 \mathrm{e}$ 50 horas de envelhecimento e uma estabilização desse valor até 400 horas. Essa redução pode ser atribuída aos mecanismos de recristalização da microestrutura e precipitação dos intermetálicos $\mathrm{Al}_{3} \mathrm{Zr}$ cúbicos $[16,18]$.

Souza [6] estudou as mesmas ligas e condições de envelhecimento que o presente trabalho, porém, sem deformação, e observou que ambas apresentaram precipitação significativa dos intermetálicos $\mathrm{Al}_{3} \mathrm{Zr}$ cúbicos nas primeiras 24 horas de envelhecimento. As condições do presente trabalho indicaram redução significativa da resistividade elétrica entre 12 e 50 horas de envelhecimento. Essa redução tanto é influenciada pela precipitação quanto pela recristalização da microestrutura, de modo que esse tempo foi maior que o observado por Souza [6].

As três condições apresentaram patamares de resistividade distintos que é atribuído ao erro experimental, tamanho reduzido das amostras e pela diferença na área da seção transversal entre as três condições.

\subsection{Microdureza}

A Figura 4 apresenta a variação da microdureza das três condições estudadas em função do tempo de envelhecimento. As amostras da liga Al-0,2\%p.Zr com 50\% de redução de altura apresentaram um aumento significativo da microdureza a partir de 50 horas de envelhecimento atingindo o pico de

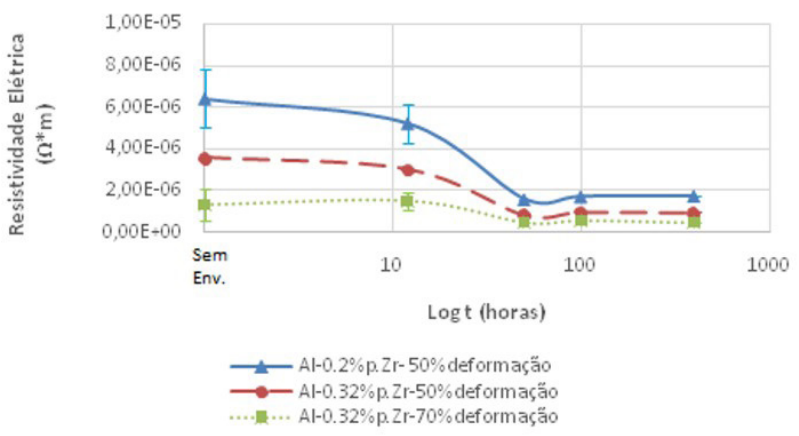

Figura 3. Variação na resistividade elétrica das ligas para condição sem envelhecimento e para envelhecimento por 12, 50, 100 e 400 horas a $377^{\circ} \mathrm{C}$.

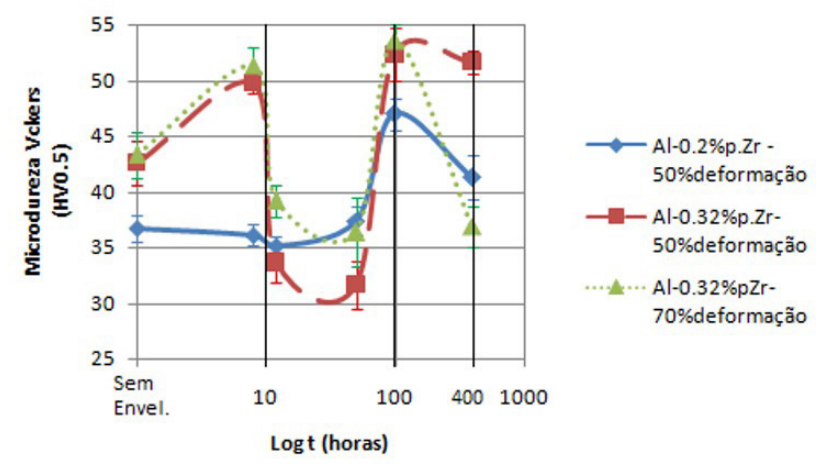

Figura 4. Variação da microdureza em função do tempo de envelhecimento a $377^{\circ} \mathrm{C}$.

endurecimento com 100 horas, entre 100 e 400 horas de tratamento essa liga apresentou redução da microdureza.

As amostras da liga Al-0,32\%p.Zr com 50 e $70 \%$ de redução de altura apresentaram comportamento semelhante. Endurecimento significativo nas primeiras 8 horas de envelhecimento, seguido de redução desse valor até 50 horas. Entre 50 e 100 horas as ligas apresentaram aumento da microdureza com pico de endurecimento em 100 horas. Entre 100 e 400 horas as ligas apresentaram redução da microdureza.

$\mathrm{O}$ aumento da microdureza da amostra da liga Al- $0,2 \%$ p.Zr com $50 \%$ de redução de altura entre 50 e 100 horas de envelhecimento é atribuído a precipitação dos intermetálicos $\mathrm{Al}_{3} \mathrm{Zr}$ cúbicos. Essa liga não apresentou redução da microdureza até 100 horas de envelhecimento, o que indica que as condições utilizadas (grau de deformação, tempo e temperatura de envelhecimento e concentração de zircônio) não foram suficientes para uma recuperação ou recristalização da microestrutura que ocasionasse redução da microdureza.

A redução da microdureza entre 100 e 400 horas é devido ao superenvelhecimento da liga que ocorre devido o 
coalescimento dos precipitados [6-9]. Esse comportamento também foi observado por Souza [6].

A liga Al-0,32\%p.Zr com 50 e 70\% de redução de altura apresentou endurecimento significativo nas primeiras 8 horas de envelhecimento devido a precipitação de intermetálicos $\mathrm{Al}_{3} \mathrm{Zr}$ cúbicos, conforme também indicado nas Figuras 1-3. Souza [6] e Du [9] também observaram precipitação significativa nas primeiras horas de envelhecimento em ligas Al-Zr.

As amostras apresentaram redução da microdureza entre 8 e 50 horas que é atribuída a recristalização da microestrutura [16,18]. Entre 50 e 100 horas as amostras apresentaram aumento da microdureza com pico de endurecimento em 100 horas que é atribuído pela continuidade da precipitação dos intermetálicos $\mathrm{Al}_{3} \mathrm{Zr}$ cúbicos que se iniciou nas primeiras 8 horas. A redução da microdureza entre 100 e 400 horas é devido o superenvelhecimento da liga, no qual há o coalescimento dos precipitados [6-9]. Souza [6] também observou esse comportamento (pico de endurecimento com 100 horas seguido de superenvelhecimento) para a liga Al-0,32\%p.Zr.

O endurecimento da liga Al-0,32\%p.Zr com 70\% de redução de altura foi um pouco maior que a mesma liga menos deformada (50\%), o superenvelhecimento (entre $100 \mathrm{e}$ 400 horas) da primeira também foi mais significativo, de modo que esse comportamento é atribuído ao maior grau de deformação aplicado (70\%) que aumentou a precipitação e, consequentemente, o endurecimento, de modo que também favoreceu um maior coalescimento dos precipitados formados.

$\mathrm{Na}$ condição sem envelhecimento as amostras da liga mais concentrada em zircônio (0,32\%p.Zr) apresentaram maior endurecimento que a liga menos concentrada em $\mathrm{Zr}$. Esse comportamento é devido ao endurecimento por solução sólida, no qual a liga com maior teor em soluto apresentou maior microdureza [6-8].

Comparando as três condições é possível observar que o aumento do teor de zircônio foi mais efetivo que a deformação no tocante ao endurecimento das ligas por precipitação, já que a liga mais concentrada em zircônio $(0,32 \%$ p.Zr) apresentou maiores valores de microdureza.

\section{Conclusão}

I. Os mecanismos de precipitação e recristalização ocorreram de forma significativa nas primeiras 50 horas de envelhecimento. Esse comportamento foi mais evidente na liga hiperperitética (0,32\%p.Zr) mais deformadas $(70 \%)$;

II. As três condições estudadas (Al- $0,2 \%$ p. Zr com $50 \%$ de redução de altura e Al-0,32\%p.Zr com 50 e $70 \%$ de redução de altura) apresentaram pico de endurecimento com 100 horas de envelhecimento a $377^{\circ} \mathrm{C}$ e redução desse valor após 400 horas. $\mathrm{O}$ endurecimento durante o envelhecimento foi devido à precipitação de intermetálicos $\mathrm{Al}_{3} \mathrm{Zr}$ de estrutura cúbica e a redução foi devido ao coalescimento desses precipitados (superenvelhecimento);

III. A deformação aplicada ocasionou uma distribuição heterogênea dos precipitados, de modo que na liga com maior deformação (70\%) esse comportamento foi mais evidente. A deformação também contribuiu para o aumento na quantidade de precipitados $\mathrm{Al}_{3} \mathrm{Zr}$ cúbicos;

IV. Após deformação e envelhecimento os precipitados $\mathrm{Al}_{3} \mathrm{Zr}$ cúbicos apresentaram formas globulares e alongadas com tamanhos variados e concentração em sítios específicos como discordâncias, bandas de cisalhamento e contornos de grão;

V. O aumento do teor de zircônio favoreceu a precipitação dos intermetálicos $\mathrm{Al}_{3} \mathrm{Zr}$ cúbicos, de modo que esse parâmetro foi mais significativo do que a deformação com relação ao endurecimento da liga.

\section{Agradecimentos}

Os autores gostariam de agradecer a CAPES e ao CNPQ pelo suporte financeiro nos anos de 2017 a 2019 e ao LCME (Laboratório Central de Microscopia Eletrônica) da Universidade Federal de Santa Catarina pelas análises laboratoriais.

\section{Referencias}

1 Zhou WW, Cai B, Li WJ, Liu ZX, Yang S. Heat-resistant Al-0.2Sc-0.04Zr electrical conductor. Materials Science and Engineering A. 2012;552:353-358. http://dx.doi.org/10.1016/j.msea.2012.05.051.

2 Mukhopadhyay AK. Microstructure and properties of high strength aluminium alloys for structural applications. Transactions of the Indian Institute of Metals. 2009;62(2):113-122.

3 Ardell AJ. Precipitation hardening. Metallurgical Transactions. A, Physical Metallurgy and Materials Science. 1985;16A:2131-2165. http://dx.doi.org/10.1007/BF02670416.

4 Knipling KE, Dunand DC, Seidman DN. Nucleation and precipitation strengthening in dilute Al-Ti and Al-Zr alloys. Metallurgical and Materials Transactions. A, Physical Metallurgy and Materials Science. 2007;38A:25222563. 
5 Knipling KE, Seidman DN, Dunand DC. Ambient and high-temperature mechanical properties of isochronally aged Al-0.06Sc, Al-0.06Zr and Al-0.06Sc-0.06Zr (at.\%) alloys. Acta Materialia. 2011;59:943-954. http://dx.doi. org/10.1016/j.actamat.2010.10.017.

6 Souza PHL. Endurecimento por precipitação em ligas de Al-Zr e Al-Zr-Mg envelhecidas artificialmente [tese]. Florianópolis: Universidade Federal de Santa Catarina; 2017.

7 Souza PHL, Oliveira CAS, Quaresma JMV. Precipitation hardening in dilute Al-Zr alloys. J Mater Res Technol. 2018;7(1):66-72. http://dx.doi.org/10.1016/j.jmrt.2017.05.006.

8 Souza PHL, Quaresma JMV, Oliveira CAS. Precipitation evolution and modeling of growth kinetics of L12-structured A13Zr Particles in Al-0.22Zr and Al-0.32Zr (wt.\%) Alloys Isothermally Aged. Materials Research. http://dx.doi.org/10.1590/1980-5373-MR-2017-0481.

9 Du X. D. Study on ageing and creep of Al-0.1Zr alloy. Materials Science and Engineering: A. 2006;432(1-2):84-89.

10 Cadirh E, Tecer H, Sahin M, Yilmaz E, Kirindi T, Gunduz M. Effect of heat treatments on the microhardness and tensile strenght of Al-0,25 wt.\% Zr alloy. Journal of Alloys and Compounds. 2015;632:229-237. http://dx.doi. org/10.1016/j.jallcom.2015.01.193.

11 Ness E. Precipitation of the metastable cubic $\mathrm{Al}_{3} \mathrm{Zr}$-phase insubperitectic $\mathrm{Al}-\mathrm{Zr}$ alloys. Acta Metallurgica. 1972;20:499-506. http://dx.doi.org/10.1016/0001-6160(72)90005-3.

12 Parameswaran VR, Weertman JR, Fine ME. Coarsening behavior of L12 phase in an Al-Zr-Ti alloy. Scripta Metallurgica. 1989;23:147-150. http://dx.doi.org/10.1016/00369748(89)90109-9.

13. Zedalis MS, Fine ME. Lattice parameter variation of Al3(Ti, V,Zr, Hf) in Al-2 at.\% (Ti, V, Zr, Hf) alloys. Scripta Metall. 1983;17(10):1247-1251. http://dx.doi.org/10.1016/0036-9748 (83) 90293-4.

14 Booth-Morrison C, Dunand DC, Seidman DN. Coarsening resistance at $400{ }^{\circ} \mathrm{C}$ of precipitation-strengthened AlZr-Sc-Er alloys. Acta Materialia. 2011;59:7029-7042. http://dx.doi.org/10.1016/j.actamat.2011.07.057.

15 3M Company. Aluminum conductor composite reinforced technical notebook. 2006 [acesso em 18 mar. 2019]. Disponível em:https://www.3m.com.br/3M/pt_BR/3m-do-brasil/todos-os-produtos-3m-do brasil/ $/$ CaboACCR/?N= $5002385+3291558271 \& \mathrm{rt}=$ rud

16 Ryum N. Precipitation and recrystallization in na Al-0.5 wt.\%Zr-alloy. Acta Metallurgica. 1969;17:269-278. http:// dx.doi.org/10.1016/0001-6160(69)90067-4.

17 El-Magd E, Abouridouane M. Characterization, modelling and simulation of deformation and fracture behaviour of the light-weight wrought alloys under high strain rate loading. International Journal of Impact Engineering. 2006;32:741-758. http://dx.doi.org/10.1016/j.ijimpeng.2005.03.008.

18 Cunha JNP, Lobato MQ, Santana CIO, Costa VS, Quaresma JMV. Influência do tratamento térmico nas propriedades elétrica e mecânica de uma liga al-fe-zr. Tecnologia em Metalurgia, Materiais e Mineração. 2018;15(2):151-158. http://dx.doi.org/10.4322/2176-1523.01302.

19 Figueiredo NC, Oliveira CAS, Masoumi M, Abreu HFG. Microstructural variations at different distance from the surface in forged $18 \mathrm{Ni} \mathrm{C300} \mathrm{maraging} \mathrm{steel.} \mathrm{Journal} \mathrm{of} \mathrm{Materials} \mathrm{Research} \mathrm{and} \mathrm{Technology.} \mathrm{2018;8(1):284-291.}$ https://doi.org/10.1016/j.jmrt.2018.01.007

20 Ünlü N. Preparation of high quality Al TEM specimens viadouble-jet electropolishing technique. Materials Characterization. 2008;59:547-553. http://dx.doi.org/10.1016/j.matchar.2007.04.003.

Recebido em: 18 Mar. 2019

Aceito em: 18 Maio 2021 